\title{
The therapeutic potential of metformin in gastric cancer
}

\author{
Sarah Courtois $^{1} \mathbb{D} \cdot$ Philippe Lehours $^{1,2} \cdot$ Emilie Bessède $^{1,2}$ \\ Received: 21 December 2018 / Accepted: 11 March 2019 / Published online: 21 March 2019 \\ (c) The International Gastric Cancer Association and The Japanese Gastric Cancer Association 2019
}

\begin{abstract}
Metformin is a biguanide molecule used since 1957 to treat type 2 diabetes patients. In addition to its hypoglycemic effects, epidemiological studies have shown that metformin can be associated with a decrease in cancer development risk in diabetic populations. Thus, since 2005 this molecule is largely studied for its antitumoural properties in different types of cancer. The potential antitumoural effect of metformin in gastric cancer has been poorly studied. Here, we detailed the different described mechanisms implicated in the antitumoural effect of metformin in gastric cancer, from the signalling pathways to the functional effects on gastric cancer cell lines and gastric cancer stem cells.
\end{abstract}

Keywords Gastric adenocarcinoma - EMT - Tumour growth · Cancer stem cells

\section{Introduction}

\section{Gastric cancer}

Gastric cancer (GC) is the fifth most common cancer in the world [1]. In 2012, a gastric adenocarcinoma was diagnosed in approximately 951,000 people and resulted in the death of 723,000 people worldwide [2]. This cancer has a poor prognosis with a 5-year survival rate of $25-30 \%$ [3], rendering GC the third leading cause of cancer-related death worldwide [1].

Current treatment is essentially based on surgery combined with conventional chemotherapies [4]. Surgical resection of the tumour can be performed at early stages when the tumour area is less than $2 \mathrm{~cm}$, without ulceration and localized in the mucosa. This surgical resection can be curative, but the majority of patients have relapses. In advanced stages, a more invasive surgery is required to remove the area where the tumour is localized, and sometimes a gastrectomy is required [4].

Sarah Courtois

courtois.sa@gmail.com

1 INSERM, Univ. Bordeaux, UMR1053 Bordeaux Research In Translational Oncology, BaRITOn, 33000 Bordeaux, France

2 French National Reference Center for Campylobacters and Helicobacters in Bordeaux (CNRCH), University Hospital of Bordeaux, Bordeaux, France
In addition to surgery, chemotherapies are used at advanced stages of GC and in relapse cases. Several clinical studies have tested different combinations of conventional chemotherapies such as ECF [epirubicin, cisplatin and 5-fluorouracil (5-FU)], ECX (epirubicin, cisplatin, capecitabine), EOX (epirubicin, oxaliplatin, capecitabine) or FLOT (fluorouracil, leucovorin, oxaliplatin, docetaxel) [5]. In patients who have not received preoperative chemotherapy, a postoperative radiotherapy or an adjuvant therapy such as paclitaxel, an antineoplastic agent, is recommended [5]. These cytotoxic chemotherapies can reduce tumour burden, but their effects are usually transient and patients may relapse. This resistance to chemotherapy is mainly supported by the cancer stem cell (CSC) population [6].

There is no specific therapy except for patients with HER2-positive tumours. Indeed, 10-15\% of GC cases display HER2 gene amplification (human epithelial growth factor receptor 2) [5] and can be treated with an anti-HER2 monoclonal antibody called trastuzumab, or Herceptin [7].

In this context, developing new efficient therapies remain necessary to improve GC care. Among the potential candidates, metformin is an interesting molecule to consider. Therefore, the aim of this review is to describe the different indications of the therapeutic potential of metformin in GC and its associated mechanisms. 


\section{Metformin, from the old anti-diabetic molecule to the promising anti-cancer drug}

Metformin is a biguanide molecule used since 1957 [8] for its hypoglycemic properties. It decreases hepatic glucose production through the suppression of gluconeogenesis, improves glucose uptake in skeletal muscles [9] and decreases resistance to insulin. In contrast with other antidiabetic molecules, metformin does not cause weight gain or increased risk of hypoglycemia. These properties made metformin the most used antidiabetic drug in type 2 diabetes treatments [10]. The exact mechanisms of action of metformin are not clearly identified. Once in the cell, metformin accumulates in the mitochondrial matrix and inhibits partially but specifically the mitochondrial respiratory chain complex I or NADH dehydrogenase [11], resulting in a reduction of ATP synthesis by ATP synthase. The increase in the ADP/ATP and AMP/ATP ratios leads to two major responses, either dependent on the activation of AMPK (AMP-activating protein kinase) or related to inhibition of cAMP synthesis [9, 11-13]. The consequences are an inhibition of expression of genes encoding specific enzymes implicated in gluconeogenesis [14], an increase in the insulin receptor IRS-2 activity, and the translocation of the receptors GLUT1 or 4 in the plasma membrane which increases glucose uptake and decreases blood glucose level [15-17]. All these elements explain the hypoglycemic effects of metformin.

In addition to its antidiabetic properties, epidemiological studies have shown that diabetic patients treated with metformin developed significantly less cancer than those not treated with metformin [18]. Evans et al. were the first to hypothesize that metformin could reduce the risk of cancer [19]. They therefore performed a pilot case-control study on diabetic patients and showed that the cancer incidence was $36.4 \%$ for diabetic patients treated with metformin, compared to $39.7 \%$ for the other diabetic patients [19]. Since then, numerous epidemiological studies have been conducted on different types of cancer. Gandini et al. performed a meta-analysis demonstrating a $31 \%$ incidence decrease and $34 \%$ mortality reduction (all cancers combined) in metformin-treated diabetic patients compared to those not treated with metformin [20]. Recently Zhou et al. published a meta-analysis of seven cohort studies including a total of 591,077 diabetic patients (type 2). According to this study, the risk of developing a GC in metformintreated diabetic patients is significantly reduced (hazard ratio of 0.76) [21]. It was also demonstrated that survival rate of GC patients is increased in the diabetic patients group treated with metformin compared to the other diabetic patients, not treated with metformin [22].
Following this finding, several publications on different types of cancer concluded that metformin possesses antitumoural properties [23-26].

\section{Direct antitumoural effects of metformin on gastric cancer}

The potential antitumoural effects of metformin in GC have been poorly studied, unlike some other types of tumours. This review summarizes the different proofs of the antitumoural effect of metformin in GC (Fig. 1).

\section{Metformin has an anti-proliferative effect on gastric cell lines}

Kato et al. were the first to study the effects of metformin in vitro and in vivo on different GC cell lines (MKN1, MKN45 and MKN74) [27]. They showed that increasing doses of metformin decreased cell proliferation in vitro. This effect was confirmed in vivo on BALB/c-nu/nu mice with subcutaneous xenografts of MKN74 cells. Mice were treated by intraperitoneal injection of 1 or $2 \mathrm{mg}$ of metformin per mouse per day during 4 weeks. At the end of the treatment, tumours were significantly smaller in the treated mice compared to the control mice (PBS). This decrease in proliferation observed in vitro and in vivo was related to a cell cycle arrest (MKN74) [27].

\section{Metformin inhibits WNT pathway through KLF5-GATA4/6- HNF4a regulation}

In 2015, Chia et al. examined copy number and gene expression of three transcription factors (KLF5, GATA4 and GATA6) in multiple GC cohorts. They showed genomic amplification and co-expression of these factors in $30 \%$ of GC [28], suggesting an important role in GC development. Indeed, a depletion of these factors using siRNAs, simultaneously or not, decreased proliferation in vitro and in vivo in YCC3 and AGS cell lines. In contrast, simultaneous overexpression of these three factors (plasmid expression) increased proliferation. KLF5 is able to interact with GATA factors. One of their direct targets is the hepatocyte nuclear factor- $4 \alpha(\mathrm{HNF} 4 \alpha)$, which is overexpressed in many types of cancer and involved in cell proliferation. When KLF5 or GATA6 were inhibited by siRNA, the expression of HNF4 $\alpha$ was significantly decreased. In addition, when the expression of $\mathrm{HNF} 4 \alpha$ was inhibited by siRNA, cell proliferation was inhibited. As HNF4 $\alpha$ expression is known to be repressed by AMPK [29], they concluded that metformin decreases the expression of HNF $4 \alpha$ and thus, decreases cell proliferation [28].

In 2016, Chang et al. analysed the transcriptomic profiles of $22 \mathrm{GC}$ tumours (compared to non-tumourous area) and 


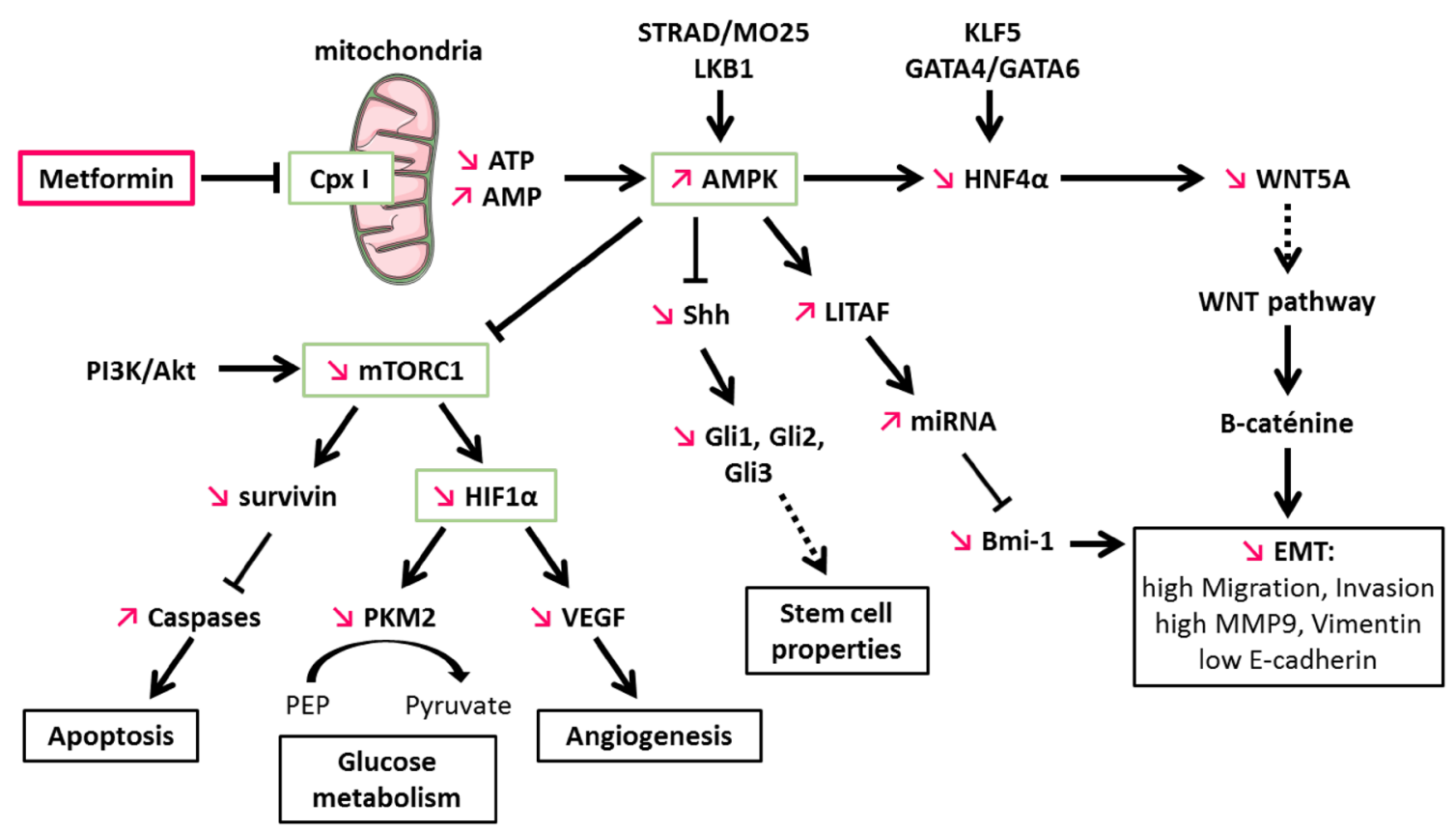

Fig. 1 Molecular mechanisms identified in response to metformin in gastric cancer model. Coloured arrows indicate demonstrated regulations induced by metformin. CPx complex I of the mitochondrial respiratory chain, EMT epithelial-to-mesenchymal transition

confirmed the overexpression of HNF4 $\alpha$ in GC. Inhibition of $\mathrm{HNF} 4 \alpha$ with shRNA or pharmacological antagonists such as BL6015 or metformin showed antineoplastic effects in vitro or in vivo on GC cell lines. Metformin activated AMPK which in turn inhibited the activity of $\mathrm{HNF} 4 \alpha$, decreased cyclins, arrested the cell cycle and inhibited tumour growth. $\mathrm{HNF} 4 \alpha$ also regulated WNT signalling through its target gene WNT5A. Indeed, when HNF4 $\alpha$ expression was inhibited, WNT5A expression was also decreased. This suggests that inhibition of $\mathrm{HNF} 4 \alpha$ inhibits the WNT pathway, known to be involved in gastric carcinogenesis [30].

These two previous studies demonstrate an interesting mechanism of action of metformin through its capacity to activate AMPK which counteracts the positive effects of KLF5/GATA4/GATA6 on the expression of HNF4 $\alpha$ and the WNT pathway thus downregulating them (Fig. 1).

\section{Metformin inhibits epithelial-to-mesenchymal transition}

The WNT/ $\beta$-catenin pathway is known to be deregulated in GC, playing a critical role in cancer development [31]. This pathway is involved in the induction of the epithelial-tomesenchymal transition (EMT) and in self-renewal properties of CSCs [32]. The previous study of Chang et al., suggested that metformin can reverse EMT in GC [30]. Valaee et al. and Jun et al. recently confirmed this effect on the GC cell line AGS $[33,34]$. Indeed, the treatment of AGS cells with metformin decreased the expression of $\beta$-catenin and vimentin [33] as well as matrix metalloproteinase-9 (MMP9) and vascular endothelial growth factor (VEGF) [34]. On the contrary, the expression of E-cadherin was increased in response to metformin [33, 34]. At functional level, metformin decreased cell viability, migration and invasive capacities [33, 35].

In addition, Huang et al. showed that metformin inhibited EMT via Bmi-1 inhibition, which is an oncogene overexpressed in many types of cancer including GC [36]. This effect was dependent on the lipopolysaccharide (LPS)-induced TNF $\alpha$ factor (LITAF), a transcription factor involved in inflammatory response. In response to lipopolysaccharides, LITAF was translocated into the nucleus where it induced the expression of different miRNAs: hsa-miR-15a, hsa-miR-194, hsa-miR-128 and hsa-miR-192 [36]. Each of these miRNAs decreased the expression of Bmi-1. Functionally, inhibition of LITAF, using shRNA, increased colony formation and cell migration, which are EMT-induced features. This study therefore presents a new axis of EMT regulation, via the activation of AMPK-LITAF-miRNA which in turn inhibits the expression of the oncogene Bmi-1 [36].

Another study lead by $\mathrm{Li}$ et al. analysed the expression levels of long noncoding RNAs (lncRNAs) via an lncRNA microarray in AGS cell line treated with metformin. They found that lncRNA H19 was greatly downregulated in the presence of metformin [35]. This lncRNA H19 is known to be overexpressed in GC tissues. The lncRNA H19 downregulation observed in the presence of metformin would be responsible for AMPK activation and the decrease of MMP9 expression. The consequences were an lncRNA 


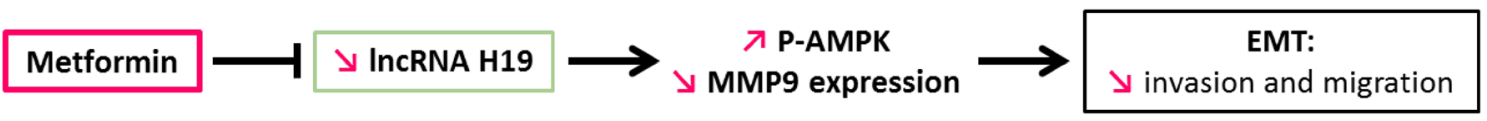

Fig. 2 lncRNA H19-dependent metformin effect. Coloured arrows indicate regulations induced by metformin. IncRNA long noncoding RNA, EMT epithelial-to-mesenchymal transition

H19-dependent decrease of cellular migration and invasion in vitro and an inhibition of tumour formation in vivo (Fig. 2). The knock-down of lncRNA H19 led to the same effect as metformin treatment. Thus, IncRNA H19 could represent a key element in the inhibition of GC cell invasion driven by metformin [35].

\section{Metformin inhibits Sonic hedgehog signalling pathway}

Song et al. have very recently demonstrated that metformin could also regulate the Sonic hedgehog (Shh) pathway. This pathway is involved in gastric carcinogenesis, its activation being essential for the maintenance of gastric CSCs characteristics (self-renewal and chemoresistance) [37]. In GC cell lines (HGC 27 and MKN 45), expression (mRNA and protein) of Shh and transcription factors Gli-1, Gli-2 and Gli-3 were decreased in response to metformin. This effect disappeared after AMPK inhibition using siRNA. Thus, metformin, via AMPK, seems to inhibit the Shh signalling pathway [38].

\section{Metformin induces apoptosis through an inhibition of survivin via mTOR pathway regulation}

In 2015, Han et al. studied the apoptotic effects of metformin in $\mathrm{GC}$ cell lines and in a non-cancerous gastric epithelial cell line (GHG-1). In GC cell lines, metformin induced expression and activation of Caspases 3 and 7 and PARP, but not in the non-cancerous cell line. This effect was combined with a decrease in survivin expression [39], a member of the inhibitor of apoptosis (IAP) family of proteins involved in apoptosis inhibition and cell cycle regulation [40]. Survivin, known to be overexpressed in many types of cancer [41], is considered as a relevant prognostic marker, including in GC [42], and represents an interesting therapeutic target. Han et al. demonstrated that metformin activates AMPK, which inhibits the mammalian Target Of Rapamycin (mTORC1) to finally inhibit survivin and induce apoptosis (Fig. 1) [39].

The mTORC1 signalling pathway integrates both intracellular and extracellular signals and regulates some central processes such as mRNA translation, lipid synthesis, glucose metabolism and autophagy, all implicated in cell growth [43]. mTORC1 is positively regulated by PI3K/Akt and MAP kinases, and negatively by AMPK [44]. mTORC1 deregulation is involved in various cellular processes such as tumour formation or angiogenesis [43] and its activation has been demonstrated in various types of cancer, including GC [45]. These observations have generated wide scientific and clinical interest for mTORC1 and hence, the increasing use of mTORC1 inhibitors such as rapamycin or its analogues (rapalogues) in the treatment of solid tumours. However, targeting mTORC1 using rapamycin has limited outcomes in patients. Recent elements highlight an additional role of mTORC1 as tumour suppressor [46], explaining the modest efficiency of rapamycin in clinical setting. Nevertheless, the ability of metformin to inhibit mTORC1 is particularly interesting and is a good indicator of its potential antitumour properties.

In GC cell lines, Chen et al. have shown that metformin decreased proliferation, induced apoptosis and decreased cell migration and invasion capabilities, which is consistent with the above studies. These effects of metformin were related to the inhibition of hypoxia inducible factor $1 \alpha$ (HIF1 $\alpha$ ) and pyruvate kinase M2 (PKM2) (mTOR effectors), which are overexpressed in advanced GC cases compared to early stages [47].

\section{Metformin is able to target gastric cancer stem cells}

Hirsch et al. were the first to study the effects of metformin on stem cell population in the breast cancer context [48]. Since then, several studies have also demonstrated anti-CSC effect of metformin in other types of cancer such as ovarian cancer [49], pancreatic [50] or colorectal cancer [51]. In GC, there is only one study that clearly and functionally demonstrates the ability of metformin to target the gastric CSCs [52]. In this study, metformin effects were evaluated on proliferation and tumourigenic properties of gastric CSCs from patient-derived primary tumour xenografts (PDXs) and GC cell lines (MKN45, AGS and MKN74). Metformin effects were evaluated in vitro in conventional two-dimensional (2D) and three-dimensional (3D) culture systems, in which only CSCs are able to form tumourspheres, and in vivo in mice xenograft models. It was shown that metformin had an anti-proliferative effect on GC cell lines in vitro in the $2 \mathrm{D}$ cultures, inducing cell cycle arrest, and in vivo with a significant decrease in the size of the remaining tumours (after subcutaneous xenograft). In 3D culture system, metformin decreased the number of tumourspheres in response to curative and preventive treatments. This effect correlated with a decrease of CSC markers expression (CD44 and Sox2) and an increase of differentiation markers expression 
(Kruppel-like factor 4 and MUC5AC). Finally, in vivo treatment of PDXs with metformin led to tumour growth delay and decreased self-renewal ability of CSCs [52].

Finally, Sekino et al. studied metformin effect in vivo on tumour growth after an intraperitoneal injection of a GC cell line (MKN45). They demonstrated that metformin treatment decreased the total tumour volume. They also showed that the remaining tumours from treated mice expressed less nuclear factor-карра B (NF- $\mathrm{BB})$ than the tumours from untreated mice [53]. NF- $\kappa B$ is known to be implicated in the EMT process through the upregulation of Snail expression [54].

These results reveal the ability of metformin to target gastric CSCs and suggest that its use could represent an efficient strategy to inhibit tumour growth and relapse.

\section{Indirect antitumoural effects of metformin on gastric cancer}

All above studies prove the direct antitumoural effect of metformin on GC cells and gastric CSCs. But, some studies investigated potential indirect effects of metformin.

\section{Metformin can modulate interaction between tumour cells and tumour microenvironment}

A recent study showed that metformin is able to modulate interaction between GC cells and their microenvironment [55]. In order to demonstrate this, they isolated gastric tumour-associated fibroblasts (TAFs) from GC patients. These TAFs were pre-treated with metformin and co-cultured with GC cells. Proliferation of GC cells co-cultured with pre-treated TAFs was decreased compared to GC cells co-cultured with non-pre-treated TAFs. Metformin treatment increased the secretion of the calmodulin-like protein 3 (Calml3) in the culture medium of gastric TAFs. Calml3 then had a suppressive effect on the clonogenicity of GC cells [55].

\section{Metformin has an immune-mediated antitumour effect}

On other cancer models, Eikawa et al. demonstrated an immune-mediated antitumour effect by metformin [56]. In vivo metformin treatment induced solid tumour rejection in mice via an activation of CD8 + tumour-infiltrating lymphocytes (TILs). Because of cancer cell persistence, TILs progressively lost their ability to secrete IL-2, TNF $\alpha$, and IFN $\gamma$ and they were finally eliminated by apoptosis in an immune exhaustion process. Metformin treatment was able to prevent TILs-apoptosis, induced TILs migration into tumours and finally reactivated antitumour immunity. This mechanism was demonstrated on different cancer models (lung, intestinal and renal carcinoma, melanoma and breast cancer) and seems through an activation of AMPK. However additional experiments are necessary to fully understand the underlying mechanism.

Finally, another interesting fact is the surprising antibiotic effect of metformin against Helicobacter pylori [57], the main risk factor of GC. Indeed, it has been recently shown in vitro and in vivo in a mouse infected-model, that metformin directly inhibited $H$. pylori growth. Furthermore, the epidemiological study performed on a Taiwanese diabetic population, suggested that metformin use was associated with a significantly reduced risk of $H$. pylori infection in a dose-response pattern [58]. These facts could indicate that metformin use decreases GC risk via its antibiotic effect against $H$. pylori.

In the same perspective, it was recently shown that metformin is able to modulate gut microbiota [59]. Hence, as it is known that microbiota can influence cancer development and progression [60], future studies could be performed to eventually demonstrate whether metformin has indirect antitumour properties via its effects on microbiota.

\section{Metformin: from bench to cancer patient's bedside}

\section{Can metformin be use alone as patient's anticancer treatment?}

Previous studies demonstrated that metformin has antitumoural properties in vitro and in vivo in mouse models. In this context the next step consists to propose a therapeutic application in oncology. Currently there is no clinical trial on GC patients, but there are phases III and IV clinical trials in other types of cancer (brain, breast, oral and prostate cancers) (Table 1). In these clinical trials, metformin is not used as a direct anticancer treatment, but more in prevention perspective to reduce cancer recurrence. These clinical trials are ongoing and no results are yet available.

\section{Can metformin be an interesting candidate to combine with conventional chemotherapies?}

In gastric cancer models, in vivo experiments were conducted by $\mathrm{Yu}$ et al. to study the effects of metformin alone or in combination with cisplatin or rapamycin (mTORC1 inhibitor) on tumour growth. Cisplatin is a conventional chemotherapy drug widely used in the treatment of various types of cancer, including GC as previously mentioned. Its anticancer activity is based on its ability to interact with purine bases, inducing the formation of intra- and interstrand DNA-protein interactions which block transcription, cellular replication and induce apoptosis [61]. Firstly, Yu et al. showed, on a subcutaneous tumour xenograft model, that metformin alone was able to decrease tumour volume and weight. Secondly, they showed, on a peritoneal tumour 


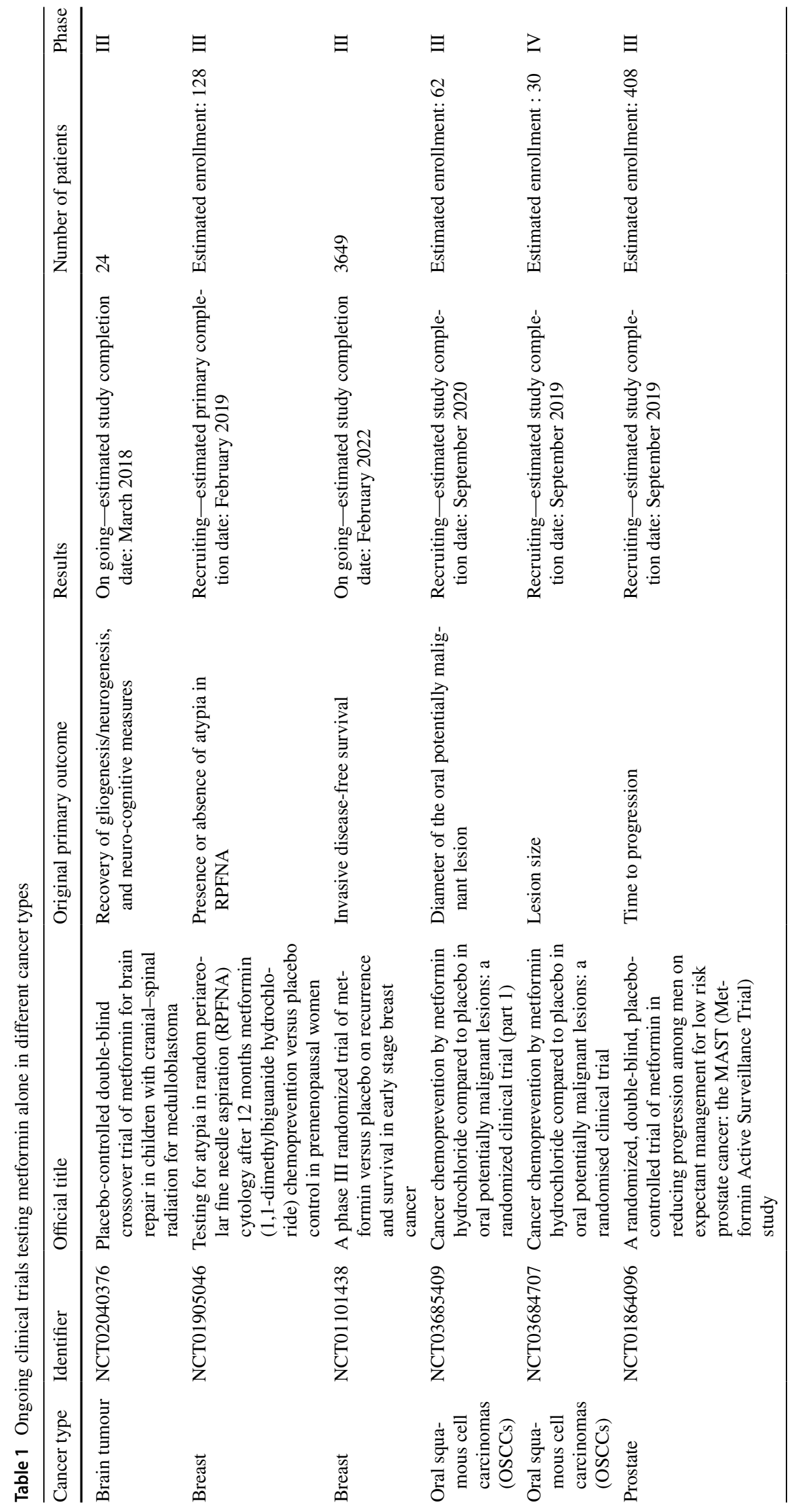




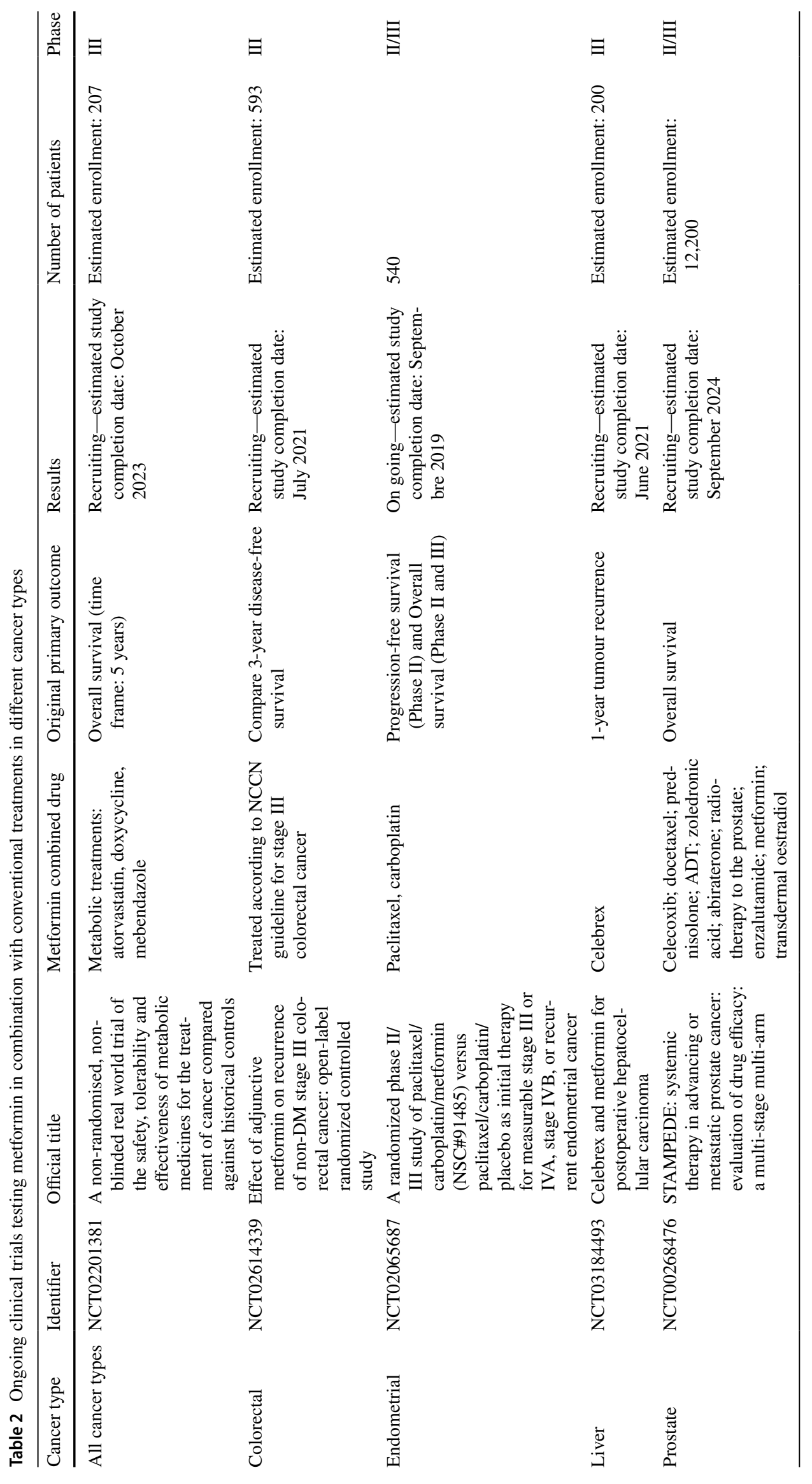


xenograft model, that the combination of metformin with cisplatin, rapamycin or both significantly inhibited peritoneal dissemination of GC and enhanced the effect of each drug alone [45]. Based on these results, the association of metformin with cisplatin could be of therapeutic interest.

However, another study of Lesan et al. raises again the question of synergy between metformin and cisplatin [62]. In their study, they showed in vitro that metformin and cisplatin separately decreased cell proliferation and induced apoptosis in the MKN45 cells. It is interesting to note that in this study, the combination of these two molecules significantly decreased the effects of cisplatin alone. They showed that survivin, mTOR and Akt expressions were increased in vitro when GC cell lines were treated with combined metformin and cisplatin, compared to a treatment with metformin alone or cisplatin alone. They proposed that this increase of survivin, mTOR and Akt expressions was responsible for the antagonist effect of metformin and cisplatin. However, these results were obtained from in vitro studies, and no in vivo study was performed [62].

Finally, Wu performed a small in vitro study on AGS cell lines showing that metformin and chemotherapeutic agents (cisplatin, adriamycin, paclitaxel) could separately decrease cell migration and invasion, and increase apoptosis. More interestingly, association of metformin with cisplatin or adriamycin or paclitaxel potentiated the effects of these drugs alone [63].

More studies are needed to confirm if, in the GC context, the association of metformin with conventional chemotherapies represents an interesting therapeutic perspective, as it is the case in hepatoblastoma for example, where metformin attenuated hypoxia-induced resistance to cisplatin in the human liver cancer cell line HepG2 [64]. This study clearly demonstrated that addition of metformin is better than conventional chemotherapy alone.

In this perspective, numerous clinical trials are on the way around the world on different types of solid cancers like breast, pancreatic, colorectal, rectal, endometrial and ovarian cancers [65]. Most of them are phase II clinical trials, moreover some are in phase III (Table 2) (https://clinicaltrials. gov/). As they are ongoing, no results are yet available. The aim of these clinical trials is to identify whether metformin combined with conventional cancer treatments can improve overall survival, progression-free survival and recurrence, compared to conventional cancer treatments alone (Table 2). For the moment, there is no clinical trial on GC patients.

\section{Conclusion}

Metformin is used since more than 50 years to treat diabetic patients and is known to be well tolerated. Furthermore, recent data have shown that metformin also has antitumoural effects in GC and regulates different signalling pathways known to be implicated in gastric carcinogenesis. Moreover, metformin can also target gastric CSCs. All these properties indicate that metformin represents a promising anti-cancer drug in GC and could lead to a better survival rate of GC patients. Its use in combination with conventional chemotherapies is being tested in clinical trials. Even if currently there is no clinical trial on GC patients, data summarized in this review suggest that it could be really interesting to use metformin to fight GC.

Acknowledgements We thank Lornella Seeneevassen, Amandine Laur and Elvire Berthenet (INSERM U1053, University of Bordeaux, Bordeaux, France) for helpful discussions.

\section{References}

1. WHO. Cancer today [Internet]. 2012. http://gco.iarc.fr/today/ home.

2. Torre LA, Bray F, Siegel RL, Ferlay J, Lortet-Tieulent J, Jemal A. Global cancer statistics, 2012. CA Cancer J Clin. 2015;65:87-108.

3. Fock KM. Review article: the epidemiology and prevention of gastric cancer. Aliment Pharmacol Ther. 2014;40:250-60.

4. Ajani JA, D'Amico TA, Almhanna K, Bentrem DJ, Chao J, Das P, et al. Gastric cancer, version 3.2016, NCCN clinical practice guidelines in oncology. J Natl Compr Cancer Netw JNCCN. 2016;14:1286-312.

5. Smyth EC, Verheij M, Allum W, Cunningham D, Cervantes A, Arnold D, et al. Gastric cancer: ESMO clinical practice guidelines for diagnosis, treatment and follow-up. Ann Oncol Off J Eur Soc Med Oncol. 2016;27:v38-49.

6. Reya T, Morrison SJ, Clarke MF, Weissman IL. Stem cells, cancer, and cancer stem cells. Nature. 2001;414:105-11.

7. Hu B, El Hajj N, Sittler S, Lammert N, Barnes R, Meloni-Ehrig A. Gastric cancer: classification, histology and application of molecular pathology. J Gastrointest Oncol. 2012;3:251-61.

8. Bailey CJ. Metformin: historical overview. Diabetologia. 2017.

9. Zhou G, Myers R, Li Y, Chen Y, Shen X, Fenyk-Melody J, et al. Role of AMP-activated protein kinase in mechanism of metformin action. J Clin Invest. 2001;108:1167-74.

10. Ferrannini E. The target of metformin in type 2 diabetes. $\mathrm{N}$ Engl J Med. 2014;371:1547-8.

11. El-Mir MY, Nogueira V, Fontaine E, Avéret N, Rigoulet M, Leverve $\mathrm{X}$. Dimethylbiguanide inhibits cell respiration via an indirect effect targeted on the respiratory chain complex I. J Biol Chem. 2000;275:223-8.

12. Madiraju AK, Erion DM, Rahimi Y, Zhang X-M, Braddock DT, Albright RA, et al. Metformin suppresses gluconeogenesis by inhibiting mitochondrial glycerophosphate dehydrogenase. Nature. 2014;510:542-6.

13. Foretz M, Guigas B, Bertrand L, Pollak M, Viollet B. Metformin: from mechanisms of action to therapies. Cell Metab. 2014;20:953-66.

14. Foretz M, Hébrard S, Leclerc J, Zarrinpashneh E, Soty M, Mithieux G, et al. Metformin inhibits hepatic gluconeogenesis in mice independently of the LKB1/AMPK pathway via a decrease in hepatic energy state. J Clin Invest. 2010;120:2355-69.

15. Gunton JE, Delhanty PJD, Takahashi S-I, Baxter RC. Metformin rapidly increases insulin receptor activation in human liver and signals preferentially through insulin-receptor substrate-2. J Clin Endocrinol Metab. 2003;88:1323-32. 
16. Ha J, Guan K-L, Kim J. AMPK and autophagy in glucose/glycogen metabolism. Mol Aspects Med. 2015;46:46-62.

17. Fischer Y, Thomas J, Rösen P, Kammermeier H. Action of metformin on glucose transport and glucose transporter GLUT1 and GLUT4 in heart muscle cells from healthy and diabetic rats. Endocrinology. 1995;136:412-20.

18. Decensi A, Puntoni M, Goodwin P, Cazzaniga M, Gennari A, Bonanni B, et al. Metformin and cancer risk in diabetic patients: a systematic review and meta-analysis. Cancer Prev Res Phila Pa. 2010;3:1451-61.

19. Evans JMM, Donnelly LA, Emslie-Smith AM, Alessi DR, Morris AD. Metformin and reduced risk of cancer in diabetic patients. BMJ. 2005;330:1304-5.

20. Gandini S, Puntoni M, Heckman-Stoddard BM, Dunn BK, Ford L, DeCensi A, et al. Metformin and cancer risk and mortality: a systematic review and meta-analysis taking into account biases and confounders. Cancer Prev Res Phila Pa. 2014;7:867-85.

21. Zhou X-L, Xue W-H, Ding X-F, Li L-F, Dou M-M, Zhang W-J, et al. Association between metformin and the risk of gastric cancer in patients with type 2 diabetes mellitus: a meta-analysis of cohort studies. Oncotarget. 2017;8:55622-31.

22. Lee C, Jung M, Jung I, Heo SJ, Jeong YH, An JY, et al. Cumulative metformin use and its impact on survival in gastric cancer patients after gastrectomy. Ann Surg. 2016;263:96-102.

23. Dowling RJO, Niraula S, Stambolic V, Goodwin PJ. Metformin in cancer: translational challenges. J Mol Endocrinol. 2012;48:R31-43.

24. Del Barco S, Vazquez-Martin A, Cufí S, Oliveras-Ferraros C, Bosch-Barrera J, Joven J, et al. Metformin: multi-faceted protection against cancer. Oncotarget. 2011;2:896-917.

25. Martin-Castillo B, Vazquez-Martin A, Oliveras-Ferraros C, Menendez JA. Metformin and cancer: doses, mechanisms and the dandelion and hormetic phenomena. Cell Cycle Georget Tex. 2010;9:1057-64.

26. Emami Riedmaier A, Fisel P, Nies AT, Schaeffeler E, Schwab M. Metformin and cancer: from the old medicine cabinet to pharmacological pitfalls and prospects. Trends Pharmacol Sci. 2013;34:126-35.

27. Kato K, Gong J, Iwama H, Kitanaka A, Tani J, Miyoshi H, et al. The antidiabetic drug metformin inhibits gastric cancer cell proliferation in vitro and in vivo. Mol Cancer Ther. 2012;11:549-60.

28. Chia N-Y, Deng N, Das K, Huang D, Hu L, Zhu Y, et al. Regulatory crosstalk between lineage-survival oncogenes KLF5, GATA4 and GATA6 cooperatively promotes gastric cancer development. Gut. 2015;64:707-19.

29. Hong YH, Varanasi US, Yang W, Leff T. AMP-activated protein kinase regulates HNF4alpha transcriptional activity by inhibiting dimer formation and decreasing protein stability. J Biol Chem. 2003;278:27495-501.

30. Chang HR, Nam S, Kook M-C, Kim K-T, Liu X, Yao H, et al. HNF4 $\alpha$ is a therapeutic target that links AMPK to WNT signalling in early-stage gastric cancer. Gut. 2016;65:19-32.

31. Chiurillo MA. Role of the Wnt/ $\beta$-catenin pathway in gastric cancer: an in-depth literature review. World J Exp Med. 2015;5:84-102.

32. Li G, Su Q, Liu H, Wang D, Zhang W, Lu Z, et al. Frizzled7 promotes epithelial-to-mesenchymal transition and stemness via activating canonical $\mathrm{Wnt} / \beta$-catenin pathway in gastric cancer. Int J Biol Sci. 2018;14:280-93.

33. Valaee S, Yaghoobi MM, Shamsara M. Metformin inhibits gastric cancer cells metastatic traits through suppression of epithelialmesenchymal transition in a glucose-independent manner. PloS One. 2017;12:e0174486.

34. Jun K-H, Lee JE, Kim SH, Jung J-H, Choi H-J, Kim YI, et al. Clinicopathological significance of $\mathrm{N}$-cadherin and VEGF in advanced gastric cancer brain metastasis and the effects of metformin in preclinical models. Oncol Rep. 2015;34:2047-53.

35. Li P, Tong L, Song Y, Sun J, Shi J, Wu Z, et al. Long noncoding RNA H19 participates in metformin-mediated inhibition of gastric cancer cell invasion. J Cell Physiol. 2019;234:4515-4527.

36. Huang D, He X, Zou J, Guo P, Jiang S, Lv N, et al. Negative regulation of Bmi-1 by AMPK and implication in cancer progression. Oncotarget. 2016;7:6188-200.

37. Song Z, Yue W, Wei B, Wang N, Li T, Guan L, et al. Sonic hedgehog pathway is essential for maintenance of cancer stem-like cells in human gastric cancer. PloS One. 2011;6:e17687.

38. Song Z, Wei B, Lu C, Huang X, Li P, Chen L. Metformin suppresses the expression of Sonic hedgehog in gastric cancer cells. Mol Med Rep. 2017;15:1909-15.

39. Han G, Gong H, Wang Y, Guo S, Liu K. AMPK/mTOR-mediated inhibition of survivin partly contributes to metformin-induced apoptosis in human gastric cancer cell. Cancer Biol Ther. 2015;16:77-87.

40. Ryan BM, O’Donovan N, Duffy MJ. Survivin: a new target for anti-cancer therapy. Cancer Treat Rev. 2009;35:553-62.

41. Jaiswal PK, Goel A, Mittal RD. Survivin. A molecular biomarker in cancer. Indian J Med Res. 2015;141:389-97.

42. Liu JL, Gao W, Kang QM, Zhang XJ, Yang SG. Prognostic value of survivin in patients with gastric cancer: a systematic review with meta-analysis. PloS One. 2013;8:e71930.

43. Saxton RA, Sabatini DM. mTOR signaling in growth, metabolism, and disease. Cell. 2017;168:960-76.

44. Laplante M, Sabatini DM. mTOR signaling at a glance. J Cell Sci. 2009;122:3589-94.

45. Yu G, Fang W, Xia T, Chen Y, Gao Y, Jiao X, et al. Metformin potentiates rapamycin and cisplatin in gastric cancer in mice. Oncotarget. 2015;6:12748-62.

46. Villar VH, Nguyen TL, Terés S, Bodineau C, Durán RV. Escaping mTOR inhibition for cancer therapy: tumor suppressor functions of mTOR. Mol Cell Oncol. 2017;4:e1297284.

47. Chen G, Feng W, Zhang S, Bian K, Yang Y, Fang C, et al. Metformin inhibits gastric cancer via the inhibition of HIF1 $\alpha / \mathrm{PKM} 2$ signaling. Am J Cancer Res. 2015;5:1423-34.

48. Hirsch HA, Iliopoulos D, Tsichlis PN, Struhl K. Metformin selectively targets cancer stem cells, and acts together with chemotherapy to block tumor growth and prolong remission. Cancer Res. 2009;69:7507-11.

49. Shank JJ, Yang K, Ghannam J, Cabrera L, Johnston CJ, Reynolds RK, et al. Metformin targets ovarian cancer stem cells in vitro and in vivo. Gynecol Oncol. 2012;127:390-7.

50. Bao B, Wang Z, Ali S, Ahmad A, Azmi AS, Sarkar SH, et al. Metformin inhibits cell proliferation, migration and invasion by attenuating CSC function mediated by deregulating miRNAs in pancreatic cancer cells. Cancer Prev Res Phila Pa. 2012;5:355-64.

51. Zhang Y, Guan M, Zheng Z, Zhang Q, Gao F, Xue Y. Effects of metformin on CD133+ colorectal cancer cells in diabetic patients. PloS One. 2013;8:e81264.

52. Courtois S, Durán RV, Giraud J, Sifré E, Izotte J, Mégraud F, et al. Metformin targets gastric cancer stem cells. Eur J Cancer. 2017;84:193-201.

53. Sekino N, Kano M, Matsumoto Y, Sakata H, Murakami K, Toyozumi T, et al. The antitumor effects of metformin on gastric cancer in vitro and on peritoneal metastasis. Anticancer Res. 2018;38:6263-9.

54. Lamouille S, Xu J, Derynck R. Molecular mechanisms of epithelial-mesenchymal transition. Nat Rev Mol Cell Biol. 2014;15:178-96.

55. Chen G, Yu C, Tang Z, Liu S, An F, Zhu J, et al. Metformin suppresses gastric cancer progression through calmodulin-like protein 3 secreted from tumor-associated fibroblasts. Oncol Rep. 2018. 
56. Eikawa S, Nishida M, Mizukami S, Yamazaki C, Nakayama E, Udono H. Immune-mediated antitumor effect by type 2 diabetes drug, metformin. Proc Natl Acad Sci USA. 2015;112:1809-14.

57. Courtois S, Bénéjat L, Izotte J, Mégraud F, Varon C, Lehours P, et al. Metformin can inhibit Helicobacter pylori growth. Future Microbiol. 2018;13:1575-1583.

58. Tseng C-H. Metformin reduces gastric cancer risk in patients with type 2 diabetes mellitus. Aging. 2016;8:1636-49.

59. Wu H, Esteve E, Tremaroli V, Khan MT, Caesar R, ManneråsHolm L, et al. Metformin alters the gut microbiome of individuals with treatment-naive type 2 diabetes, contributing to the therapeutic effects of the drug. Nat Med. 2017;23:850-8.

60. Garrett WS. Cancer and the microbiota. Science. 2015;348:80-6.

61. Dasari S, Tchounwou PB. Cisplatin in cancer therapy: molecular mechanisms of action. Eur J Pharmacol. 2014;740:364-78.

62. Lesan V, Ghaffari SH, Salaramoli J, Heidari M, Rostami M, Alimoghaddam K, et al. Evaluation of antagonistic effects of metformin with cisplatin in gastric cancer cells. Int J Hematol Oncol Stem Cell Res. 2014;8:12-9.

63. Wu X. Effect of metformin combined with chemotherapeutic agents on gastric cancer cell line AGS. Pak J Pharm Sci. 2017;30:1833-6.

64. Fujita H, Hirose K, Sato M, Fujioka I, Fujita T, Aoki M, et al. Metformin attenuates hypoxia-induced resistance to cisplatin in the HepG2 cell line. Oncol Lett. 2019;17:2431-40.

65. Zhang H-H, Guo X-L. Combinational strategies of metformin and chemotherapy in cancers. Cancer Chemother Pharmacol. 2016;78:13-26.

Publisher's Note Springer Nature remains neutral with regard to jurisdictional claims in published maps and institutional affiliations. 